\title{
Tratamento Psicológico Frente às Representações Sociais dos Médicos
}

\author{
Psychological Treatment Faced with Social Representations of Physicians
}

\section{Tratamiento Psicológico Frente a las Representaciones Sociales de los Médicos}

Wandina Soares da Silva ${ }^{1}$, Leconte de Lisle Coelho Junior ${ }^{2}$

${ }^{1}$ Curso de Psicologia/Centro Universitário Uninassau de Campina Grande, Brasil, Email: wandinasilva@gmail.com, Orcid: https://orcid.org/0000-0002-0279-1921

${ }^{2}$ Curso de Psicologia/Centro Universitário Uninassau de Campina Grande, Brasil, Email: lecontecoelho@gmail.com, Orcid: https://orcid.org/0000-0002-9451-3303

\section{ARTICLE INFO}

Article history:

Received 2021-05-13

Accepted 2021-06-24

Available online 2021-06-25
Palavras-chave: Médicos. Representações sociais. Tratamento psicológico. Psicologia da Saúde.

Keywords: Physicians. Social representations. Psychological treatment. Psychology of Health.

Palabras-clave: Médicos. Representaciones Sociales. Tratamiento Psicológico. Psicología de la salud.

RESUMO. As representações sociais apresentam a imagem de um grupo sobre um objeto de estudo. O presente trabalho tem como objetivo: Entender a percepção dos médicos sobre o tratamento psicológico. É um estudo de campo, qualitativo e descritivo. O estudo foi realizado com dez médicos numa clínica médica na cidade de Campina Grande - Paraíba. Os instrumentos de coleta foram compostos por: questionário sociodemográfico, técnica de associação livre de palavras e entrevista semiestruturada. $\mathrm{Na}$ análise dos dados foi utilizado o software Iramuteq específico para dados textuais. Observou-se a percepção dos médicos em variadas temáticas. Para estes o tratamento psicológico auxilia no combate ao excesso de medicação. Compreendem o trabalho do psicólogo como interventivo e humanizado. Os médicos percebem que a relação do paciente com a patologia está ligada aos aspectos emocionais, ambientais e sociais, assim apontaram que encaminham pacientes para tratamento psicológico por diversos fatores não apenas transtornos mentais.

ABSTRACT. The social representations present the image of a group on an object of study. The present work aims to: Understand the perception of the doctors about the psychological treatment. It is a study, qualitative and descriptive. The study was done out with ten physicians in a medical clinic in Campina Grande - Paraíba. The collection instruments were composed of: sociodemographic questionnaire, free association technique and semi-structured interview. In the analysis of the data, Iramuteq software was used specific for textual data. The physicians' perceptions were observed in various subjects. For these the psychological treatment helps in the fight against the excess of medication. They understand the psychologist's work as intervenient and humanized. The physicians perceive that the patient's relationship with the pathology 
is linked to the emotional, environmental and social aspects, so they pointed out that they refer patients for psychological treatment for several factors, not just mental disorders.

RESUMEN. Las Representaciones sociales presentan la imagen de un grupo sobre un objeto de estúdio. El presente trabajo tiene como objetivo: comprender la percepción de los médicos sobre el tratamiento psicológico. És un estudio de campo, cualitativa y descriptiva. El estudio fue realizado con diez médicos en Campina Grande - Paraíba. En los instrumentos de recogida fueron compuestos por: cuestionário sociodemográfico, técnica de asociación libre de palabras y entrevista semiestructurada. En el análisis de los datos se utilizó el software Iramuteq específico para datos textuales. Se observaron la percepción de los médicos en variadas temáticas. Para estos, el tratamiento psicológico ayuda en el combate al exceso de medicación. Comprenden el trabajo del psicólogo como interventivo y humanizado. Los médicos perciben que la relación del paciente con la patología está ligada a los aspectos emocionales, ambientales y sociales, así señalaron que orientan pacientes para tratamiento psicológico por diversos factores no sólo transtornos mentales.

\section{Introdução}

A psicologia visa auxiliar o indivíduo e a sociedade no tocante ao comportamento e estilo de vida saudáveis, contribuindo para melhor qualidade de vida, esta adentra no contexto da saúde proporcionando eficácia enquanto ciência e profissão aponta a relação entre comportamento e saúde e comportamento e doença. A efetividade da psicologia é percebida pelos profissionais de saúde e usuários através do tratamento psicológico, o fazer do psicólogo é realizado nas intervenções que objetiva prevenir doenças e auxiliar no manejo ou no enfrentamento destas (ALMEIDA; MALAGRIS, 2011).

Conforme o Conselho Federal de Psicologia (2008), esta profissão possui um leque de diversidade no que diz respeito aos serviços que pode oferecer à população brasileira. Um serviço em qualquer área, como a psicologia escolar, psicologia organizacional ou psicologia hospitalar pode ser tido como 'tratamento' já que tal ciência se insere na dimensão das ciências da saúde. De tal forma que 'tratamento' não é um serviço relacionado exclusivamente à psicologia clínica.

\section{Fundamentação}

No documento "Atribuições Profissionais do Psicólogo no Brasil" de 2013, a palavra 'tratamento' aparece apenas na dimensão explicativa da psicologia clínica. No entanto, esmiuçando as demais descrições tem-se na página 6 em referência ao psicólogo escolar que:

Desenvolve, com os participantes do trabalho escolar (pais, alunos, diretores, professores, técnicos, pessoal administrativo), atividades visando a prevenir, identificar e resolver problemas psicossociais que possam bloquear, na escola, o desenvolvimento de potencialidades, a auto-realização e o exercício da cidadania consciente (BRASIL, 2013).

Isto é, um trabalho desenvolvido aparentemente à médio ou longo prazo que pode ser definido também como 'acolhimento' ou 'acompanhamento' (MICHAELIS, 
2016, p. 790). Outro exemplo, observando Carvalho (2013) em seu debate sobre a variedade da psicologia hospitalar, identifica-se uma extensa ramificação de saberes que se de um lado se confrontam de outro se complementam, todos em direção a uma melhor adequação do tratar o bem-estar da clientela. Tratamento, portanto, não diz respeito efetivamente à um processo terapêutico, isto é, modelo clássico e biomédico (Ribeiro, 2011).

Profissionais da psicologia atuam em diversos campos não apenas clínico (CONSELHO FEDERAL DE PSICOLOGIA, 2013; MORAIS; MORAIS; REIS; KOLLER, 2009). O trabalho desse profissional nos serviços públicos aumenta cada vez mais e o entendimento deste no setor da saúde é motivador para o desenvolvimento da psicologia como profissão. Desse modo é coerente atualizar-se quanto às novas percepções ou quais mudaram no tocante às atribuições do profissional de psicologia, até mesmo, o que isso implica para o desenvolvimento das intervenções psicológicas (LIMA; SOUZA FILHO; OLIVEIRA, 2006).

Referenciando 0 trabalho do psicólogo, vale ressaltar sua ênfase multidisciplinar. Desse modo, como apontam Miranda-Sá Junior (2013) e Ribeiro (2011) o aumento da busca por tratamento psicológico pode, então, ser relacionado com a prática médica. Isto decorre de vários sentidos, tais como os médicos visando obter conhecimento acerca das intervenções psicológicas, ou mesmo os leigos tentando compreender o que seria a psicologia (LEME; BUSSAB; OTTA, 1989; SOUZA FILHO; OLIVEIRA; LIMA, 2006).

Nos novos campos de saúde, como por exemplo: Unidades Básicas de Saúde ou Consultórios de Rua é ampliada a atuação do psicólogo ao exemplo de intervenções para prevenção de enfermidades (MORAIS; MORAIS; REIS; KOLLER, 2009). Assim é necessário um manejo adequado do profissional de psicologia para atender as demandas apresentadas nos setores públicos e coletivos.

Nesta direção, o apoio psicológico no tratamento de doenças crônicas, ao exemplo de pacientes com câncer, tem destacado a eficácia desse trabalho em junção com o serviço médico, visto que patologias podem ocasionar ansiedade e sofrimento psíquico, seus aspectos subjetivos influenciam na própria dinâmica ao engajamento terapêutico (SILVA; ROCHA; VANDENBERGHE, 2009).

Isto quer dizer que o estado psicológico de uma pessoa implica nos aspectos do processo saúde-doença (ALMEIDA; MALAGRIS, 2011; RIBEIRO, 2011). Neste sentido é possível acreditar nas vantagens para ambas às profissões que tratam do mesmo paciente, sendo este não apenas um paciente, mas um humano que necessita ser visualizado com olhar humanizado e assim construir novas perspectivas quanto ao processo patológico, viabilizando o bem-estar e melhor qualidade de vida. Desse modo a relação do paciente ao seu sintoma, o modo de se estruturar e traçar estratégias de enfrentamento pode articular a integralidade das ciências que tem o objetivo em comum de posicionar os sujeitos frente à doença (MANGIA et al., 2017).

Diante disso, por não haver remissão de sintomas em doenças crônicas e sim tratamento, faz-se necessário à junção da terapêutica psicológica com a terapêutica médica e a compreensão destes é esclarecedor. De acordo Linck, Bielemann e Lange (2008) a respeito de doenças crônicas, por exemplo, apontam que o impacto causado pelas enfermidades crônicas na economia mundial em 2020 chega aos $65 \%$ das despesas de saúde.

Cerca de 50\% de pessoas com doenças crônicas nos países desenvolvidos não aderiram, significativamente, à alguma conduta terapêutica, entre elas, tratamentos psicológicos, ao se levantar o impacto negativo tanto para o doente quanto para o Estado (LINCK, BIELEMANN; LANGE, 2008). Para compreender o que 
representam os médicos sobre o tratamento psicológico, foi escolhida a Teoria das Representações Sociais de Serge Moscovici.

\subsection{As Representações Sociais}

As representações sociais são fundamentais na compreensão de objetos sociais e sujeito na sociedade, além de sua relação com o meio social. Portanto, representar algo, é reproduzi-lo, comunicar e observar a realidade social em função de compromisso com esta (MOSCOVICI, 2003).

A teoria das representações sociais é importante pelos achados para a ciência psicológica e pesquisas sociais. Seu relevante contributo é o entendimento da relação sujeito e sociedade, internalização subjetiva e externalidade, universalidade e particularidade e a ênfase no caráter dinâmico da produção do conhecimento. $O$ sujeito e o objeto 'reapresentado' não são distintos, são inseparáveis, sendo um conjunto que se revelam na formação das representações sociais (FLORES et al., 2014, SHIMIZU et al., 2015).

Nesse sentido tratando sobre conhecimento, o teórico das representações sociais Serge Moscovici abre o caminho para a ciência participar do senso comum, contribuindo para transformar a realidade social, através da produção científica e do compromisso social entre os pares.

Além disso, Moscovici (2012) não fez distinção entre psicologia social psicológica e sociológica, pois sua teoria tem a perspectiva de entender a relação das formações imagéticas e ideológicas opostas na produção da imagem aglutinadora de grupos, e o diálogo destas, na consolidação das interações sociais.

Para o autor, é necessário conhecer o social para pesquisar a psicologia social. Todo pensamento e compreensão têm as Representações Sociais como base envolvido no processo de interação e comunicação das pessoas, elas transformam conhecimentos científicos em conhecimento de senso comum. Compreende-se a objetividade através da ciência, assim como se compreende o pensamento unânime pelas representações sociais num grupo, ou seja, pelo conjunto de conceitos explicados e afirmados no cotidiano (FLORES et al., 2014).

A Teoria das Representações Sociais engloba os termos da objetivação e ancoragem. A objetivação é uma condição de deixar concreto aquilo que era abstrato, objetivando a imagem social de grupos. Por sua vez, a ancoragem consiste nas atribuições dadas aos objetos das relações sociais, ou seja, algo que parece estranho a um grupo torna-se comum pela categorização desse objeto (MOSCOVICI, 2012).

Nesse contexto, em virtude da coletividade é relevante levantar características grupais sobre o tratamento psicológico, para isso adentra-se na Teoria das Representações Sociais que busca elucidar a imagem produzida pelo grupo dos médicos sobre o que para eles seja o tratamento psicológico.

Espera-se que este estudo leve a melhor compreensão de como o papel da psicologia é percebido pelos médicos. Verificar como as pessoas percebem uma prática profissional se faz necessário em qualquer área de atuação, possibilitando o reconhecimento de aspectos positivos, negativos, vantagens e limitações dessa prática (SOUZA FILHO; OLIVEIRA; LIMA, 2006).

Para tanto, esta pesquisa teve como objetivo, entender as representações sociais dos médicos sobre o tratamento, identificando o impacto do acompanhamento psicológico para o tratamento dos seus pacientes e descrevendo de que modo a psicologia contribui para saúde na concepção da amostra. 


\section{Metodologia}

\subsection{Tipo de Estudo}

Esse estudo é caracterizado como uma pesquisa de campo de metodologia qualitativa e descritiva.

3.2 Amostra

A princípio foram entrevistados dois médicos como pesquisa piloto para preparação posterior. A estruturação da amostra foi de conveniência composta por médicas e médicos que atendem em uma clínica autorizada para o estudo, sendo constituída por dez (10) entrevistados. A clínica em questão é um centro médico e dispõe de ampla quantidade de médicos com diferentes especialidades.

\subsection{Instrumentos}

Os instrumentos que foram utilizados: um questionário sociodemográfico e um roteiro de entrevista semiestruturado; afora isso, a técnica de associação livre de palavras (TALP). Esta última, é uma técnica oral é baseada em achados de testes projetivos, o qual o sujeito utiliza da memorização para revelar conteúdos psíquicos (COUTINHO; DO BÚ, 2017). O questionário sociodemográfico trouxe questões que permitiram traça o perfil da amostra, como se verá mais adiante.

Conforme Flick (2004), a pesquisa de modelo qualitativo se sustenta nas entrevistas por que ao inverso dos modelos experimentais, as pessoas têm uma maior espontaneidade para expressar aquilo que pensam, sentem e também suas experiências. Deste modo, tal modelo foi escolhido justamente para que se ficasse claro quais imagens e mesmo discursos estão se constituindo sobre o tratamento psicológico neste grupo de profissionais.

\subsection{Procedimentos de Coleta de Dados}

Quanto aos procedimentos de realização da pesquisa, foram realizadas de forma individual, no consultório de cada médico. Estes foram comunicados pela gerência da instituição antecipadamente. Foi combinado aos que aceitaram participar da pesquisa, um melhor horário, no início ou final do expediente. $O$ pesquisador ajustou-se ao horário conforme a disponibilidade do participante. Portanto, a pesquisa foi realizada entre o mês de setembro e outubro de 2018. Os mesmos receberam o termo de consentimento livre esclarecido, foram apresentados os objetivos da pesquisa e todos os esclarecimentos. A entrevistadora apresentou de forma sucinta 0 tema do projeto. Cada participante foi orientado a respeito dos instrumentos de coleta de dados e aspectos éticos.

Referente aos aspectos éticos a pesquisa está regulada pelas Diretrizes e Normas de Pesquisa em Seres Humanos, através da Resolução №. 466/12 do Conselho Nacional de Saúde do Ministério da Saúde. A pesquisa tem a autorização de Comitê de Ética com o Número do CAAE: 91382218.5.0000.5175. Foi utilizado o termo de Consentimento Livre Esclarecido - TCLE formalizando o consentimento dos participantes da amostra, ocorrendo assim à informação legal destes quanto à decisão de participação na pesquisa. Estes aspectos éticos clarificaram aos participantes 0 direito de possível desistência da pesquisa conforme desejassem. Assim, sob caráter sigiloso foi assegurado à confidencialidade dos dados obtidos.

\subsection{Análise dos Dados}

Por continuidade, os processamentos de análise dos dados se deram na verificação das respostas coletadas, sendo utilizado o IRAMUTEQ - software específico para análise de dados textuais. Esse tipo de programa informativo tem significância quanto às pesquisas em ciências humanas e sociais. Desta forma, Flick (2004, p.22) indica que o modelo de pesquisa qualitativo: 
“(...) estuda o conhecimento e a práticas dos participantes. (...) as interrelações são descritas no contexto concreto do caso e explicadas em relação a este. A pesquisa qualitativa considera que pontos de vista e práticas no campo são diferentes devido às diversas perspectivas subjetivas e ambientes sociais a eles relacionados".

Por isso, Camargo e Justo (2013) destacam a relevância sobre os estudos não somente da instância simbólica, mas também do campo imagético-condutual especificado em relação ao contexto social. Este programa analisa o texto e o subdivide em etapas, fornecendo a classificação hierárquica das palavras e aponta uma nuvem de palavras que demonstra a recorrência das mais destacadas na fala dos entrevistados.

\section{Resultados e Discussões}

Os dados sociodemográficos podem ser observados detalhadamente na Tabela 1. Dos 10 participantes integrantes deste estudo, a maioria (60\%) é do sexo masculino e o restante do sexo feminino. O intervalo de idades possui amplitude de 28-50 e possui como média: 35,9 anos. E o desvio padrão, $D P=37.9$. No que se refere ao estado civil, $80 \%$ dos participantes são casados (a) e o restante são solteiros (as).

Tabela 1 - Distribuição dos Dados Sociodemográficos dos Médicos $(\mathrm{n}=10)$

\begin{tabular}{|l|c|}
\hline Variáveis & $\%$ \\
\hline Sexo & 60 \\
\hline Masculino & 40 \\
\hline Estadino civil & \\
\hline Casados & 80 \\
\hline Solteiros & 20 \\
\hline Religião & \\
\hline Católica & 50 \\
\hline Espirita & 20 \\
\hline Não declarou & 30 \\
\hline Especialidade & \\
\hline Clinico geral & 10 \\
\hline Cardiologia & 10 \\
\hline Dermatologia & 20 \\
\hline Endocrinologia & 10 \\
\hline Neurologia & 10 \\
\hline Otorrinolaringologia & 10 \\
\hline Pediatria & 10 \\
\hline Psiquiatria & 10 \\
\hline Reumatologia & 10 \\
\hline Níveis de atenção à saúde & \\
\hline Primário & 20 \\
\hline Secundário & 20 \\
\hline Terciário & 60 \\
\hline Distribuição por níveis & \\
\hline Um nível & 10 \\
\hline Dois níveis & 60 \\
\hline Três níveis & 30 \\
\hline Formação: Minnmo de 5 anos & $\mathbf{2}$ \\
\hline
\end{tabular}

Tempo de Formação: Mínimo de 5 anos, Máximo 23 anos

Idade (28-50) $M=35,9$ e $D P=37,9$

Fonte: Os autores (2021) 
A religião predominante é a católica (50\%), a religião espírita aparece com $20 \%$ e $30 \%$ destes não declarou religião. Na distribuição da especialidade médica, a distribuição se deu da seguinte forma: neurologista (1); psiquiatra (1); pediatra (1); clínico geral (1); endocrinologista (1); otorrinolaringologista (1); reumatologia (1); cardiologista (1) e dermatologista (2). Os médicos que participaram da pesquisa atuam em diferentes níveis de atenção à saúde, sendo estes níveis primário, secundário e terciário, como visto na tabela acima.

A seguir será apresentada a análise pelo método 'nuvem de palavras' a partir do software IRAMUTEQ. Conforme alguns autores (ANDRADE JUNIOR; ANDRADE, 2016; CAMARGO; JUSTO, 2013), o programa cria uma imagem similar a uma nuvem com palavras agrupadas e estruturadas apresentadas em diversos tamanhos. As palavras maiores são as que tiveram maior frequência, sendo assim permitindo uma rápida identificação das palavras-chaves do 'corpus'.

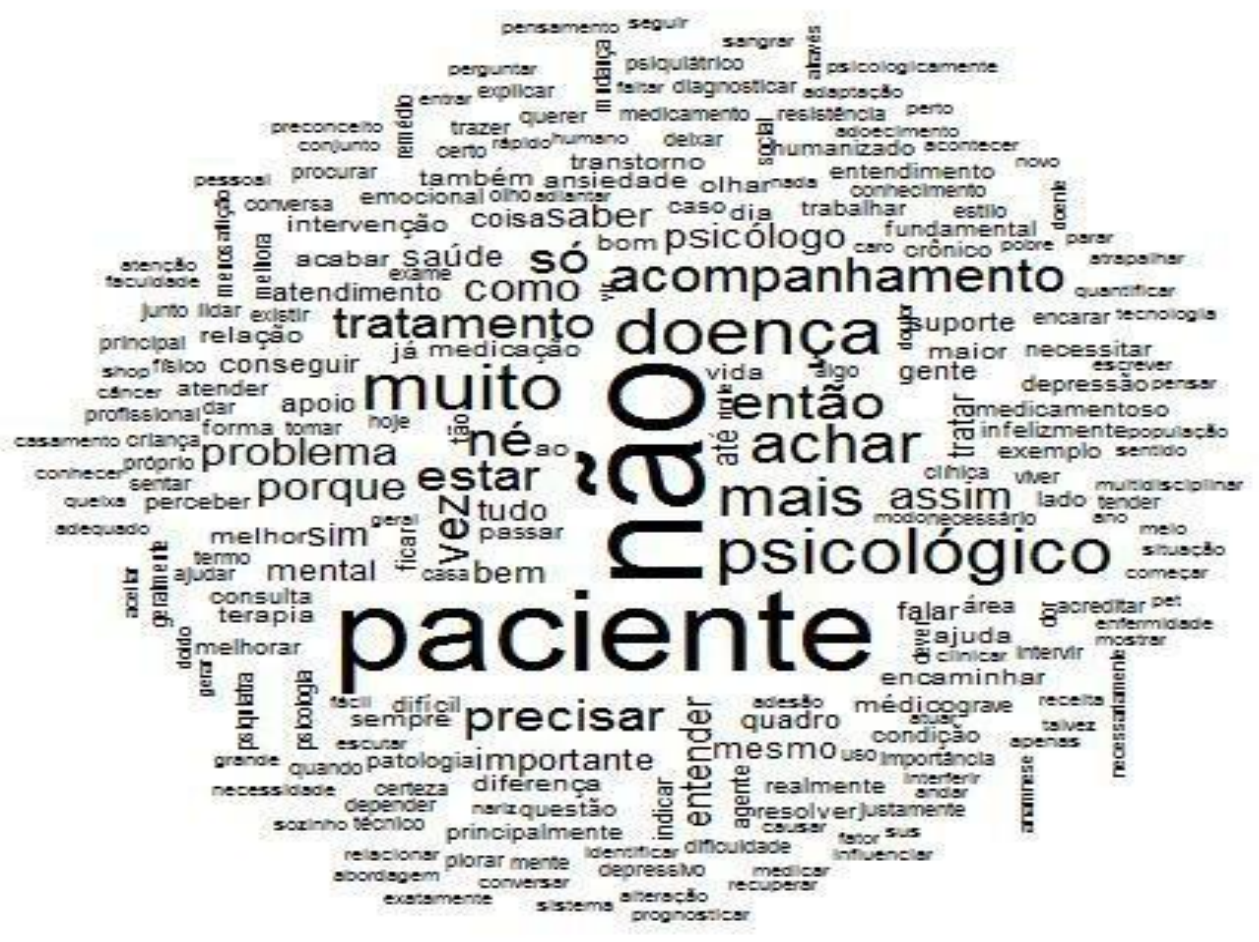

Figura 1. Nuvem de palavras com as Respostas dos médicos do Roteiro de Entrevista. Campina Grande-PB, Brasil, 2018

Fonte: Os autores (2021)

Através da nuvem é possível observar a prevalência de palavras no eixo central: Paciente, acompanhamento, doença, psicológico, tratamento, problema; essas palavras remetem às mais frequentes no corpus do texto que por sua vez são as palavras mais utilizadas pelos médicos na resposta do roteiro de entrevista, a frequência aponta significância da evocação a respeito destas palavras (CAMARGO; JUSTO, 2013).

As palavras intermediárias ficam nas extremidades da nuvem, são elas: Psiquiátricos, conversa, diagnosticar, explicar, atendimento, humanizado, transtorno, emocional, medicamento; estas palavras são menos enfatizadas na fala dos 
entrevistados. Nesse sentido, através do roteiro de entrevista percebe-se a diversidade do conceito médico sobre tratamento psicológico, e o quanto isso se distancia pelo apresentado por Miranda-Sá Junior (2013), por exemplo.

Tabela 2 - Análise de Classificação Hierárquica Descendente (CHD) das Respostas dos Entrevistados $(\mathrm{n}=10)$

\begin{tabular}{|c|c|c|c|c|c|c|c|}
\hline \multicolumn{2}{|c|}{ CLASSE 2} & CLASSE 1 & & \multicolumn{2}{|c|}{ CLASSE 3} & CLASSE 4 & \\
\hline $33,7 \%$ & & $25,6 \%$ & & $26,6 \%$ & & $14,1 \%$ & \\
\hline Palavra & $\mathrm{X}^{2}$ & Palavra & $\mathrm{X}^{2}$ & Palavra & $\mathbf{X}^{2}$ & Palavra & $X^{2}$ \\
\hline Intervenção & 18 & Dificuldade & 17 & Doença & 17 & Doenças & 26 \\
\hline Exame & 10 & Necessidade & 17 & Grave & 14 & Emocionais & 12 \\
\hline Técnica & 8 & Psicológico & 14 & Problema & 9 & Psiquismo & 12 \\
\hline Humanizado & 7 & Acompanhamento & 12 & Psiquiátrico & 8 & Criança & 12 \\
\hline Clínica & 6 & Adesão & 11 & Aflição & 5 & Relacionamentos & 12 \\
\hline Diagnóstico & 6 & Medicação & 9 & Sociedade & 5 & & \\
\hline
\end{tabular}

Fonte: Os autores (2021)

O dendrograma de Classificação Hierárquica Descendente (CHD) destaca os elementos presentes nas respostas dos participantes. $\mathrm{O}$ corpus textual analisado na pesquisa foi composto por 10 textos (10 respostas), que o programa repartiu em 10 segmentos de textos (Sts), que continham 383 palavras ou formas distintas que ocorreram: 13615 vezes. A análise definiu que as palavras com frequência igual ou superior a 3 e com $x 2>=3,545$ seriam válidas. A CHD reteve $77,55 \%$ do total de Sts, gerando quatro classes. Ao se reduzir os vocábulos às suas raízes, obtiveram-se 1194 lematizações, que resultaram em 1163 palavras ou formas ativas analisáveis, como se vê na figura 2 abaixo: 

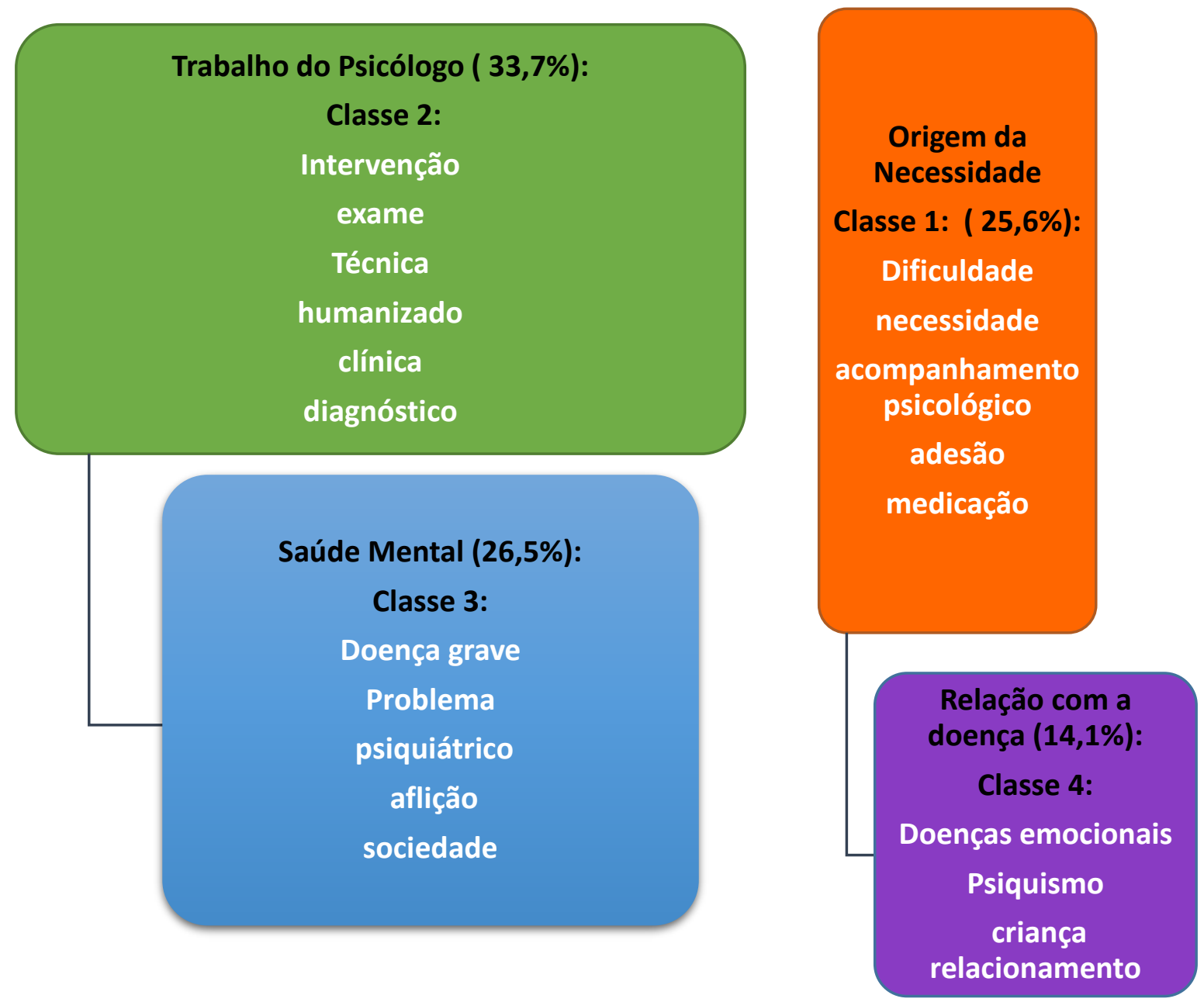

FIGURA 2 - Dendrograma de Classificação Hierárquica Descendente (CHD).

Fonte: Os autores (2021)

Pela CHD são percebidas as respostas apresentadas por categorias, observadas por classes na tabela 2. Cada classe recebe uma definição categórica. Portanto a classe 1 é responsável por $25,6 \%$ do corpus, sendo nomeada como origem da necessidade de tratamento. Esta demonstra que os médicos percebem que muitos pacientes necessitam de acompanhamento psicológico, pois, vários apresentam ansiedade ou depressão frente a sua doença, acreditam que o tratamento psicológico melhora a adesão terapêutica do próprio paciente diminuindo assim uso de medicações. Destaca-se a fala dos participantes ao mencionar a necessidade do tratamento psicológico:

"[...] no caso de pacientes com doenças crônicas eu acho necessário acompanhamento psicológico [...] "eu vejo como complementar, como qualquer outra área de saúde " "[...] os que fazem acompanhamento psicológico tendem a se conhecer melhor, eles entendem melhor a situação” (Participante 5).

"[...] pacientes com doenças crônicas, dor crônica, se algo não for feito para manter a saúde mental desse, ele vai evoluir para um paciente de saúde mental" (Participante 8). 
Tal discurso, corrobora aquilo que outros autores já haviam pesquisado: o saber psicológico embora seja útil, ainda não é compreendido e, portanto, é visto como 'complementar' (CARVALHO, 2014; SILVA; ROCHA; VANDENBERGHE, 2010). Os vocábulos da nuvem de palavras que podem se relacionar à esta classe são "acompanhamento", "tratamento" e as menos relevantes: "conversa" e "atendimento", isto é, são relacionadas ao significado de "tratamento".

A classe 2 , responsável por $33,7 \%$ do corpus, sendo essa é a maior parte, refere-se ao Trabalho do psicólogo como aquele que intervém e é habilitado com técnica humanizada, auxiliando o paciente no enfrentamento de seu diagnóstico, levando a este a partir daí o conhecimento de seu problema e situa o paciente diante disso. Nas falas abaixo se destaca a percepção do trabalho do psicólogo:

"[...] ás vezes a pessoa tá tão de depressão, tão grande que precisa de uma terapia comportamental para ter uma intervenção né? Acho que nesse caso não cabe uma psicanálise" (Participante 3).

"[...] entendo que ele vai dar aquele suporte ao paciente, mas, exatamente o meu entendimento de como isso é feito, nem as diferenças de vertentes e as modalidades dentro da psicologia [...] é uma terapia não medicamentosa do quadro do paciente" (Participante 5).

Os fragmentos dos discursos dos participantes 3 e 5 confirmam a falta de conhecimento dos processos de atendimento dos psicólogos e fortalecem a ideia de tratamento clínico, sem perceber as diversas nuances que formalizam outras áreas de tal profissão. Seu desconhecimento do que um psicólogo pode fazer além do trabalho clínico converge para aquilo que os sujeitos da pesquisa de Souza Filho, Oliveira e Lima (2006) expressaram anteriormente. Por outro lado, mesmo que não entendam as técnicas da psicologia, estes compreendem que ela dá suporte aos pacientes.

A classe 3, aponta $26,6 \%$ do corpus, indicando aspectos da saúde mental ao abordar sobre temas como psiquiátrico, doença grave, aflição; nesta classe é possível perceber na fala dos médicos que os pacientes têm consternação e sofrimento frente ao desconhecido que é simbolizada pela patologia. Isto remete ao transtorno emocional que para os informantes da pesquisa tentam elaborar como funciona 0 tratamento psicológico.

"[...] muitos pacientes ficam jogados em serem apenas dopados em quadros de transtornos mentais, tem paciente com depressão grave que usa três clonazepam no dia, dois diazepam, três fenergan, realmente você num vai sentir nada assim" (Participante 8 ).

"[...] na minha percepção é como eu disse o psicólogo ele consegue entender o que tá se passando com o paciente, ele consegue enxergar o paciente de uma forma, que a gente muitas vezes pensa na patologia né?!" (Participante 9).

Nesta classe também é interessante a fala dos participantes 8 e 9 ao dizerem que alguns transtornos mentais são causados por doenças orgânicas como é o caso da depressão e ansiedade. Os entrevistados apontam nessa modalidade que as questões da saúde mental e tratamento psiquiátrico ficam nas margens da sociedade e isso se dá por falta de investimento público, e por ser mais fácil o acesso ao medicamento os pacientes tem-Ihe a preferência. O processo de acompanhamento 
psicológico seria, portanto, o procedimento que aliviaria, por exemplo, os custos financeiros e emocionais destas pessoas pois os ajudariam em vários contextos como deixam claro alguns autores (ALMEIDA; MALAGRIS, 2011; RIBEIRO, 2011).

Ainda sobre o contexto de saúde mental e seus serviços, o Ministério da Saúde informou em 2010 que no Brasil, por volta de 23 milhões de pessoas $(12 \%$ da população) usam ou usaram, pelo menos uma vez, os serviços de saúde mental (PRADO; FRANCISCO; BARROS, 2017). Desse modo, é primordial compreender o tratamento psicológico como prevenção de comorbidades em situações de pessoas com doenças crônicas, e por sua vez corroborando no auxílio para questões de vulnerabilidade de saúde mental.

A classe 4, traz em números, $14,1 \%$ do corpus. Esta apresenta percepções sobre a relação do paciente à sua doença, e enfatizam que os fatores emocionais desencadeiam algumas doenças, os aspectos psicológicos da pessoa que está doente andam junto com o seu processo de adoecimento.

"[...] encarar as doenças nem maior do que elas são, nem menor do que elas são a gente tem que encarar as doenças do tamanho que elas são. se você encarar a doença maior do que ela é, ela vai lhe trazer problema que não é da doença, talvez um problema psicológico" (Participante 2).

"[...] muitas vezes se sentem depressivos pela própria doença, se sente com medo de morte, com medo de progressão da doença, porque são doenças progressivas que incapacita entendeu?!, E eles ficam assim depressivos por isso" (Participante 3).

Percebe-se que a doença do paciente pode leva-lo a outras patologias, desse modo à psicologia atua como multidisciplinar favorecendo o paciente quando esta busca o serviço, a fala a seguir representa a importância do tratamento psicológico:

"[...] porque sempre eu digo que o tratamento psicológico é importante, porque só medicação eu não acredito que faça, medicação ajuda com certeza, mas, precisa ter um conjunto a psicoterapia, o psicólogo, mas, infelizmente o que pesa é o fator cultural, é muito forte, infelizmente" (participante 3).

Os médicos da pesquisa percebem o benefício do tratamento psicológico, interpretando como auxiliar no caráter multidisciplinar com a medicina. Ocorrem ganhos a respeito da intervenção psicológica, e isto reduz a procura por consumo medicamentoso e aumenta aderência à terapêutica que favorece a mudança de comportamento e novo estilo de vida. Essas mudanças não se baseiam apenas em relação aos transtornos mentais, mas, várias outras patologias como por exemplo: doenças cardiovasculares, doenças oncológicas, artrite reumatoide, entre outras (CARVALHO, 2014).

"[...] é fundamental porque acho que muitas enfermidades precisam de um acompanhamento psicológico, tem doenças gravíssimas que se a pessoa tiver abalada emocionalmente ela piora e muito, $o$ paciente que faz acompanhamento psicológico ele é muito melhor" (participante 10).

Carvalho (2014) analisando o estado emocional nas pessoas com patologias, observou a diferença entre pacientes atendidos apenas por médico e, pacientes que em junção com o médico eram atendidos também por psicólogos. A autora verificou 
que a melhoria do estado emocional geral é encontrada em $80 \%$ dos casos seguidos por psicólogos, enquanto a melhoria no estado emocional é menos citada em 50,4\% de pacientes atendidos apenas pelo médico.

Os resultados apontaram ainda que todos entrevistados encaminham pacientes para tratamento psicológico, porém alguns deles mencionam que mesmo indicando o tratamento percebem em vários pacientes que existe resistência para isso e acreditam que ocorrem por fatores culturais, econômicos, idade e falta de conhecimento sobre a psicologia. Afirmam que pacientes se apoiam numa antiga objetivação de representação social de que "psicólogo é para doido" (LEME, BUSSAB; OTTA, 1989). Os participantes 2, 3 e 7 comentam:

"[...] tem muito paciente que eu digo; olha isso aí num é só comigo não. Você precisa de um acompanhamento psicológico, 'não doutor, mas eu num sou doido não!'” (Participante 2).

"[...] porque infelizmente criou-se essa cultura né?! Que quem trata de doido é psicólogo, aí infelizmente né essa imagem negativa atrapalha né?!" (Participante 3).

"[...] precisam de três, quatro consultas até mais por mês, então eles não procuram porque a maioria não tem condições de fazer, os que fazem têm uma melhora muito mais rápida e o prognóstico muito melhor do que os que não fazem" (Participante 7).

No que diz respeito às representações sociais, neste estudo os resultados convergem para quatro (4) classes que as formam: origem da necessidade de tratamento, trabalho do psicólogo, saúde mental, e, relação do paciente à sua doença. Conforme Moscovici (2012) na sua constituição nuclear, as representações guardam as objetivações e ancoragens para a formulação da imagem aglutinadora do grupo no que tange à um tema que produz interação social entre os membros de um mesmo agrupamento ou destes em relação a outros.

Embora a visão da medicina sobre a psicologia seja distante e mesmo deturpada enquanto modelo científico (MIRANDA-SÁ JUNIOR, 2013). No entanto, a classe, 'O trabalho do psicólogo' é a que mais condensa conhecimento pela prática do dia-a-dia cada vez mais supõe-se que estes profissionais ao menos tentam entender o que seja a psicologia.

A forma de trabalho psicológico, embora ainda voltado ao modelo biomédico, ou seja, a compreensão do que faz o psicólogo se concretiza através do tratamento propriamente dito (sua práxis clínica, neste caso). Isto é o núcleo das representações que permite que os médicos reconheçam os psicólogos como pares da mesma área de trabalho, ou seja, a saúde.

Este componente imagético por sua vez se ancora em outras duas classes a saber: 'A origem da necessidade de tratamento' e a 'Saúde mental'. Para a primeira há um forte entrelaçamento, pois, o trabalho do psicólogo, que é o tratamento, se relaciona, como em qualquer trabalho da área de saúde a um processo etiológico. $\mathrm{E}$ a classe saúde mental é justamente um dos âmbitos mais relevantes em que a psicologia pode contribuir. Trabalhar com a saúde mental como visto nos discursos apresentados faz parte da função dos profissionais de psicologia, não somente no tratamento, mas principalmente na prevenção e promoção à saúde (RIBEIRO, 2011).

Por fim, a classe 'relação paciente à sua doença', perfaz a representação social, pois aqui a amostra pesquisada identifica a conexão que existe entre sua 
própria clientela e aquilo que os faz ser tornarem clientes, isto é, as enfermidades, seja de ordem física ou mental. Portanto, compreende-se que a representação social sobre o tratamento psicológico é de eficácia, mas, estes não conhecem a psicologia em profundidade e talvez por não terem mais informações sobre a atuação do psicólogo e os possíveis tratamentos psicológicos de forma efetiva, estes não compreendam ao certo os benefícios pontuais que a psicologia desenvolve com seu trabalho teórico e técnico. Desse modo seria oportuno e eficaz trabalho de psicoeducação a respeito da terapêutica psicológica com o público dos profissionais da medicina, entendendo que o conhecimento os levará à pratica de solicitar os serviços de psicologia com mais precisão.

\section{Considerações Finais}

A pesquisa objetivou compreender as representações sociais dos médicos sobre o tratamento psicológico, que por sua vez, se manifestou através dos discursos dos participantes. Remetendo a compreensão do tratamento psicológico como essencial tanto em casos de transtornos mentais quanto em auxilio no tratamento de pacientes de diversas doenças, visto que em geral as pessoas precisam de apoio psicológico.

Portanto, para estes a psicologia traz efeitos terapêuticos, dinâmicos, flexíveis e coadjuvantes a saúde. Desse modo, é possível compreender pelo estudo em questão que o objetivo geral proposto foi alcançado. As representações sociais se assentam em quatro classes que versam sobre a origem da necessidade do tratamento, o suposto trabalho dos psicólogos e psicólogas, a saúde mental como área de atuação e relação que os pacientes possuem com a doença acometida. Os objetivos específicos foram corroborados, o impacto do acompanhamento nos pacientes é superficial pois não houve argumento que mostrasse que efetivamente estes médicos encaminhassem seus pacientes e que os mesmos tiveram alguma melhora no tratamento. Por outro lado, os discursos acusam que a psicologia pode ser um eficiente instrumento de apoio emocional concomitante ao tratamento medicamentoso.

Sugere-se que mais estudos sejam efetuados sobre esta temática a fim de corroborar ou não com pesquisas longitudinais, os achados expostos neste texto, pois, a presente pesquisa destaca limitações como pequena amostra pela conveniência e curto período de tempo do estudo.

\section{Referências}

ALMEIDA, Raquel A.; MALAGRIS, Luciana Emanoel N. A prática da psicologia da saúde. Revista da SBPH, v. 14, n. 2, p. 183-202, 2011. doi: http://pepsic.bvsalud.org/scielo.php?script=sci arttext\&pid=S1516-

$08582011000200012 \& \operatorname{lng}=\mathrm{pt \& t}$ Ing=pt. $\quad$ Disponível

em

http://pepsic.bvsalud.org/scielo.php?script=sci abstract\&pid=S1516-

$08582011000200012 \& \mathrm{ng}=\mathrm{pt \& nrm}=\mathrm{iso}$. Acesso em 17 fev 2018.

ANDRADE JUNIOR., Edson O., \& ANDRADE, Edson O. Lexical analysis of the Code of Medical Ethics of the Federal Council of Medicine. Revista da Associação Médica Brasileira, v. 62, n. 2, p. 123-130, 2016. doi: https://dx.doi.org/10.1590/18069282.62.02.123. Disponível em https://www.scielo.br/j/ramb/a/Qgyw8nLRC9H7zWW8kjKvNwg/abstract/?lang=en . Acesso em 30 mai 2018.

CAMARGO, Brigido V.; JUSTO, Ana Maria. IRAMUTEQ: um software gratuito para análise de dados textuais. Temas em Psicologia, v. 21, n. 2, p. 513-518, 2103. doi: http://pepsic.bvsalud.org/scielo.php?script=sci_arttext\&pid=S1413-

389X2013000200016\&lng=pt. http://dx.doi.org/10.9788/TP2013.2-16. Disponível em 
http://pepsic.bvsalud.org/scielo.php?script=sci arttext\&pid=S1413-

389X2013000200016. Acesso em 29 mai 2018.

CARVALHO, Denis B. Psicologia da saúde crítica no contexto hospitalar. Psicologia ciência e profissão. v. $33, \quad$ n. $2, \quad$ p. 350-365, 2013. doi: https://dx.doi.org/10.1590/S1414-98932013000200008. Disponível em https://www.scielo.br/j/pcp/a/MnXKpCyJbNTLrgxmxci5fWR/abstract/?lang=pt.

Acesso em 27 mai 2018.

CARVALHO, Silvia. Psicoterapia e Medicina Geral e Familiar: o potencial da terapia cognitivo comportamental. Revista Portuguesa de Medicina Geral e Familiar, v. 30, n. 6, p. 406-409, $2014 . \quad$ doi: http://www.scielo.mec.pt/scielo.php?script=sci arttext\&pid=S2182-

$51732014000600010 \&$ lng=pt\&tlng=pt. $\quad$ Disponível em https://www.rpmgf.pt/ojs/index.php/rpmgf/article/view/11406. Acesso em 01 jun 2017. CONSELHO FEDERAL DE PSICOLOGIA. Atribuições Profissionais do Psicólogo no Brasil. Disponível em https://site.cfp.org.br/wpcontent/uploads/2008/08/atr_prof_psicologo_cbo.pdf. Acesso em 18 out. 2018.

COUTINHO, Maria da Penha L.; Do BÚ, Emerson A. A técnica de associação livre de palavras sobre o prisma do software tri-deux-mots (version 5.2). Revista Campo do Saber, v. 3, n.1): 219-243, 2017. doi: Retrivied from http:// periodicos.iesp.edu.br/index.php/campodosaber/ article/view/72/58. Disponível em https://periodicos.iesp.edu.br/index.php/campodosaber/article/view/72. Acesso em 01 jun 2018.

FLICK, Uwe. Uma introdução à pesquisa qualitativa. Porto Alegre: Bookman, 2004.

FLORES, Talita Meireles et al. Considerações sobre a Teoria das Representações Sociais como capítulo da História da Psicologia Social. Bol. - Acad. Paul. Psicol., São Paulo, v. 34, n. 87, p. 320-335, dez. 2014. Disponível em <http://pepsic.bvsalud.org/scielo.php?script=sci_arttext\&pid=S1415-

711X2014000200003\&lng=pt\&nrm=iso >. Acesso em 23 jun. 2021.

LEME, Maria A. Vanzolini. S.; BUSSAB, Vera S. Raad; OTTA, Ema. A representação social da Psicologia e do psicólogo. Psicologia: Ciência e Profissão, v. 9, n. 1, p. 2935, 1989. doi: https://dx.doi.org/10.1590/S1414-98931989000100009. Disponível em https://www.scielo.br/j/pcp/a/xDhhK9mHwQShaF9NRsmZzqG/?lang=pt

Acesso em 01 fev 2018.

LINCK, Caroline L.; BIELEMANN, Valquíria L. M.; SOUSA, Afra S.; LANGE, Celmira Paciente crônico frente ao adoecer e a aderência ao tratamento. Acta Paulista de Enfermagem, v. 21, n. 2, p. 317-22, 2008. doi: http://www.scielo.br/pdf/ape/v21n2/pt a14v21n2.pdf. Disponível em https://www.scielo.br/j/ape/a/MnDd3S3Tmp7Dszkmr6fMvZs/?lang=pt\&format=pdf Acesso em 25 jul 2018.

MANGIA, Ariella Sebastião et al. Quais fatores clínicos, funcionais e psicológicos antes do tratamento são preditores de má qualidade de vida em pacientes com câncer no final da quimioterapia? Revista da Associação Médica Brasileira [online], v. 11: 978987, 2017. doi: ISSN 0104-4230. http://dx.doi.org/10.1590/1806-9282.63.11.978. Disponível em https://www.scielo.br/j/ramb/a/7GtqS4m4v7fsD6RM99NBGNm/?lang=en\&format=pdf. Acesso em 12 jul 2018.

MICHAELIS, Henriette. Dicionário escolar da língua portuguesa. São Paulo: Melhoramentos, 2016.

BRASIL. Ministério do Trabalho. Catálogo Brasileiro de Ocupações. Psicologia. Brasília: MT, 2013. 
MIRANDA-SÁ JUNIOR, Luis S. Uma introdução à medicina. O médico. Brasília: CFM, 2013.

MORAIS, Normanda A.; MORAIS, Camila A.; REIS, Silvia; KOLLER, Silvia H. Promoção de saúde e adolescência: um exemplo de intervenção com adolescentes em situação de rua. Psicologia \& Sociedade, v. 22, n. 3, p. 507-518, 2010. doi: https://dx.doi.org/10.1590/S0102-71822010000300023. Disponível em https://www.scielo.br/j/psoc/a/kxpKCFF7z3z3aPJxBg8RZGp/abstract/?lang=pt.

Acesso em 13 jul 2018.

MOSCOVICI, Serge. Representações Sociais: investigação em Psicologia Social. Petrópolis: Vozes, 2013.

MOSCOVICl, Serge. A psicanálise, sua imagem e seu público. Petrópolis: Vozes 2012.

PRADO, Maria A. M. B.; FRANCISCO, Priscila M. S. B.; BARROS, Marilisa B. A. Uso de medicamentos psicotrópicos em adultos e idosos residentes em Campinas, São Paulo: um estudo transversal de base populacional, Epidemiologia e Serviços de Saúde, v. 26, n.4, p. 747-758, 2017. doi: https://dx.doi.org/10.5123/s167949742017000400007. Disponível em https://www.scielo.br/j/psoc/a/kxpKCFF7z3z3aPJxBg8RZGp/abstract/?lang=pt.

Acesso em 20 jan 2018.

RIBEIRO, José P. A psicologia da saúde. In: Alves, Railda F. (Org.). Psicologia da Saúde: Teoria, intervenção e pesquisa (pp. 23-64). Campina Grande: Eduepb, 2011. SHIMIZU, Helena E.; REIS e SILVA, Jéssica; MOURA, Luciana de Melo; BERMÚDEZ, Ximena P. A estrutura das representações sociais sobre saúde e doença entre membros de movimentos sociais. Ciência \& Saúde Coletiva [online]. v. 20, n. 9, p. 2899-2910, 2015. doi: ISSN 1413-8123. http://dx.doi.org/10.1590/141381232015209.20592014. Disponível em https://www.scielo.br///csc/a/rC8Q6M8zdZjJ7kmkVP9vbtk/?format=html. Acesso em 30 jul 2018.

SILVA, Daiane S.; ROCHA, Eliana P.; VANDENBERGHE, Luc. Tratamento psicológico em grupo para dor crônica. Temas de Psicologia, v. 18, n.2, p. 335-343, 2010. doi: http://pepsic.bvsalud.org/scielo.php?script=sci arttext\&pid=S1413389X2010000200008\&lng=pt\&tlng=pt. Disponível em http://pepsic.bvsalud.org/scielo.php?script=sci arttext\&pid=S1413389X2010000200008. Acesso em 01 out 2018.

SOUZA FILHO, Marcílio L.; OLIVEIRA, Josevânia S. C.; LIMA, Flávio Lúcio A. Como as pessoas percebem o psicólogo: um estudo exploratório. Paidéia, v. 16, n. 34, p. 253-261, 2006. doi: https://dx.doi.org/10.1590/S0103-863X2006000200013. Disponível em https://www.scielo.br///paideia/a/vRGfq9yw8rPhhh9xqnBQ8hP/abstract/?lang=pt. Acesso em 14 de mar 2018. 